\title{
4-week profile of pain at movement after painful day surgery: a prospective cohort study
}

\author{
N. Belkaid, J. Dubois, H. Jalil, L. Jamaer, J-P. Ory, B. Stessel \\ Department of Anaesthesia and Intensive Care Unit, Jessa Hospital, Hasselt, Belgium
}

\section{Background and goal of the study}

Motivated by economical motives, many governments encourage to perform more complex and painful surgical procedures in an ambulatory setting. Adequate pain treatment at home however is a principal endpoint after day surgery An optimal characterization of the acute pain trajectory over the first postoperative month after painful surgical procedures is a prerequisite for the development of future preventive, procedure-specific pain-treatment schedules. Therefore, we aimed to assess and compare 4-week pain profiles of 4 painful day surgery procedures.

\section{Methods}

We analyzed data from a cohort of patients who participated in a randomized trial investigating postoperative pain medication. Two hundred patients undergoing elective hemorrhoid surgery $(n=50)$, arthroscopic shoulder $(n=50)$ or knee surgery $(n=50)$, or unilateral inguinal hernia repair $(n=50)$ in day setting were enrolled. All patients undergoing shoulder surgery received an interscalenic block. All patients were treated with paracetamol, an NSAID or metamizole and tramadol as needed during the first 4 postoperative days. Pain medication therapy after this period was subscribed by the surgeon. Postoperative pain intensity at movement was assessed at postoperative day 0, 1, 2, 3,4,7,14 and 28 using a Numeric Rating Scale (NRS) by telephone call.

\section{Results and Discussion}

Four patients were excluded from analysis: three patients underwent a bilateral inguinal hernia repair and one patient was immediately excluded because of excessive preoperative opioid use.
During the first 3 POD's, 5 patients were lost-to-follow-up. From POD 3 tot POD 28, another 19 patients were lost-tofollow-up. Mean pain at movement scores per type of surgery are presented in figure 1. Arthroscopic shoulder surgery was found to be most painful over the whole trajectory. Inguinal hernia repair and arthroscopic knee surgery were least painful.

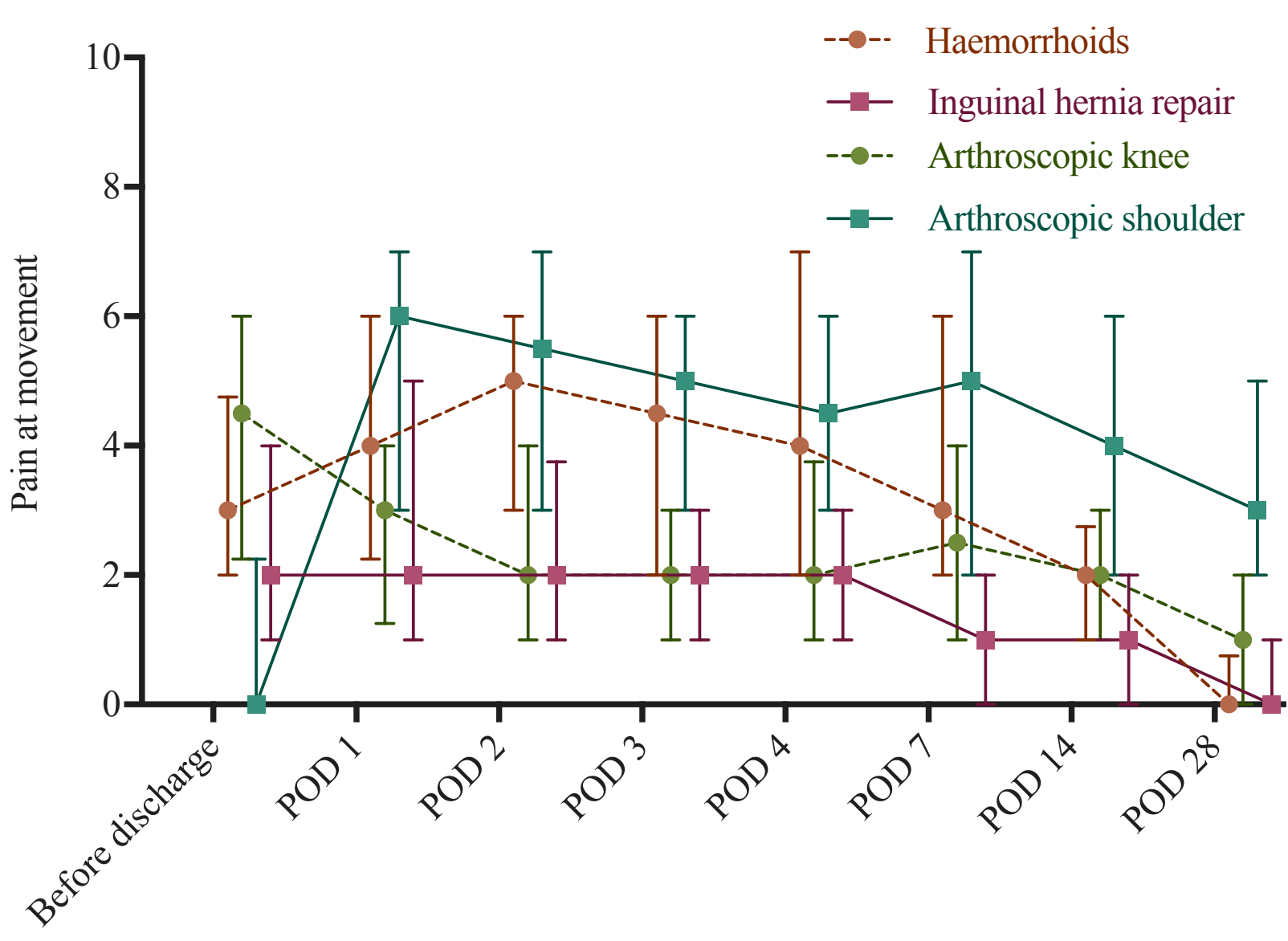

Figure 1: Pain at movement. Y-as: NRS $0-10 . P O D=$ postoperative day.

\section{Conclusion}

Our results suggest that each type of day surgery has its own unique postoperative pain trajectory. New strategies for pain therapy at home should be developed, in particular after ambulatory arthroscopic shoulder surgery and hemorrhoid surgery. 